\title{
Land use and land cover mapping using rule-based classification in Karbala city, Iraq
}

\begin{abstract}
Land use and land cover are important and useful geographic information system (GIS) layers that have been used for a wide range of geospatial applications. These layers are usually generated by applying digital image processing steps for a satellite image or images captured from an aircraft. Several methods are available in literature to produce such GIS layers. Image classification is the main method that has been used by many researchers to produce thematic maps. In the current study, a decision tree was used to develop rulesets at object level. These rules were applied and a thematic map of Karbala city was produced from SPOT image. The overall accuracy of the classification image was $96 \%$ and the kappa index was 0.95 . The results indicated that the proposed classification method is effective and can produce promising results.
\end{abstract}

Keyword: Land use/cover; Decision tree; GIS; Remote sensing 